Recibido: 24/09/2021 --- Aceptado: 10/11/2021 --- Publicado: 10/12/2021

\title{
SISTEMAS DE VERIFICACIÓN EN MEDIOS NATIVOS DIGITALES E IMPLICACIÓN DE LA AUDIENCIA EN LA LUCHA CONTRA LA DESINFORMACIÓN EN EL MODELO IBÉRICO
}

\section{VERIFICATION SYSTEMS IN DIGITAL NATIVE MEDIA AND AUDIENCE INVOLVEMENT IN THE FIGHT AGAINST DISINFORMATION IN THE IBERIAN MODEL}

\author{
D José Sixto-García ${ }^{1}$. Universidade de Santiago de Compostela (USC). España \\ jose.sixto@usc.es \\ (8) Ana-Isabel Rodríguez-Vázquez. Universidade de Santiago de Compostela \\ (USC). España \\ anaisabel.rodriguez.vazquez@usc.es
}

Xosé López-García. Universidade de Santiago de Compostela (USC). España xose.lopez.garcia@usc.es

\section{Cómo citar este artículo:}

Sixto-García, J., Rodríguez-Vázquez, A-I., \& López-García, X. (2021). Sistemas de verificación en medios nativos digitales e implicación de la audiencia en la lucha contra la desinformación en el modelo ibérico. Revista de Comunicación de la SEECI, 54, 41-61. http://doi.org/10.15198/seeci.2021.54.e738

\section{RESUMEN}

La desinformación constituye una seria amenaza para el ejercicio democrático del periodismo y para las sociedades en general. Mientras la prensa se adhiere a las recomendaciones y códigos éticos de diferentes organizaciones para proteger los derechos de los ciudadanos a recibir información veraz, los medios digitales ofrecen cada vez más posibilidades de participación de la audiencia. Perteneciente a esta doble realidad, esta investigación aborda los sistemas de verificación de contenidos generados por los usuarios de los periódicos nativos digitales más importantes de España y Portugal. Para este trabajo se entrevistó a los responsables de los sistemas dentro de cada periódico. Las conclusiones señalan que los periódicos otorgan gran

\footnotetext{
${ }^{1}$ José Sixto-García. Universidade de Santiago de Compostela (USC). jose.sixto@usc.es. José SixtoGarcía es profesor del Departamento de Ciencias de la Comunicación de la Universidade de Santiago de Compostela y forma parte del grupo de investigación Novos medios. Sus intereses de investigación incluyen contextos digitales, redes sociales y nuevas narrativas y su impacto en la producción de noticias. ORCID: https://orcid.org/0000-0002-2988-0975
} 
importancia a la participación de las audiencias en la producción informativa, aunque también exponen la falta de sistemas de verificación de la información brindada por el público. Este trabajo aporta el análisis de casos concretos y la perspectiva de los profesionales en la lucha contra la desinformación. Aquí se argumenta que no es suficiente que los medios de comunicación se adhieran a pautas deontológicas externas, sino que deben desarrollar sistemas internos de verificación de noticias para recuperar su credibilidad, como fue el caso, por ejemplo, durante la pandemia de Coronavirus (ComScore, 2020).

PALABRAS CLAVE: Desinformación; comprobación de hechos; noticias falsas; ética de los medios; medios digitales nativos; democracia, audiencia.

\section{ABSTRACT}

Disinformation constitutes a serious threat to the democratic exercise of journalism and to societies in general. While the press adheres to the recommendations and ethical codes of different organizations to protect citizens' rights to receive truthful information, digital media offer more and more possibilities for audience participation. Pertaining to this dual reality, this research deals with the usergenerated contents verification systems of the most important digital native newspapers in Spain and Portugal. For this job, those responsible for the systems within each newspaper were interviewed. The conclusions indicate that newspapers attach great importance to the involvement of audiences in news production, though they also expose the lack of verification systems for the information provided by the public. This work provides the analysis of specific cases and the perspective of professionals in the fight against disinformation. It is argued here that it is not enough for the media to adhere to external deontological guidelines, but rather that they should develop internal news verification systems to regain their credibility, as was the case, for example, during the Coronavirus pandemic (ComScore, 2020).

KEYWORDS: Disinformation; fact-checking; fake news; media ethics; native digital media; democracy; audience.

\section{SISTEMAS DE VERIFICAÇÃO EM SUPORTES DIGITAIS NATIVOS E ENVOLVIMENTO DO PÚBLICO NO COMBATE À DESINFORMAÇÃO NO MODELO IBÉRICO}

\section{RESUMO}

A desinformação constitui uma séria ameaça ao exercício democrático do jornalismo e às sociedades em geral. Enquanto a imprensa adere às recomendações e códigos de ética de diferentes organizações para proteger os direitos dos cidadãos de receber informações verdadeiras, as mídias digitais oferecem cada vez mais possibilidades de participação do público. Pertencente a esta dupla realidade, esta pesquisa aborda os sistemas de verificação de conteúdos gerados pelos utilizadores dos mais importantes jornais digitais nativos de Espanha e Portugal. Para este trabalho, foram entrevistados os responsáveis pelos sistemas de cada jornal. As conclusões indicam que os jornais atribuem grande importância à participação do público na produção 
Sixto-García, J., Rodríguez-Vázquez, A-I., \& López-García, X. Sistemas de verificación en medios nativos digitales e implicación de la audiencia en la lucha contra la desinformación en el modelo ibérico.

das notícias, embora também exponham a falta de sistemas de verificação das informações prestadas pelo público. Este trabalho fornece a análise de casos específicos e a perspectiva de profissionais no combate à desinformação. Aqui, argumenta-se que não basta a mídia aderir a diretrizes éticas externas, mas que deve desenvolver sistemas internos de verificação de notícias para recuperar sua credibilidade, como foi o caso, por exemplo, durante a pandemia do Coronavírus (ComScore, 2020).

PALAVRAS CHAVE: Desinformação; verificando os fatos; notícias falsas; ética na mídia; mídia digital nativa; democracia, audiência.

Traducción por Paula González (Universidad Católica Andrés Bello, Venezuela)

\section{INTRODUCCIÓN}

El derecho de los ciudadanos a recibir información veraz se ve oscurecido por la difusión de noticias falsas y la falta de sistemas de verificación de contenido en los medios de comunicación. Esta situación de desinformación socava nuestra experiencia democrática y pone en peligro el compromiso del periodismo con la promoción de una sociedad libre y pluralista. Por un lado, los periodistas son activos en el desarrollo de opciones para incentivar la participación ciudadana e involucrar a sus audiencias en la elaboración de las noticias. Sin embargo, por otro lado, la falta de filtros permite que se publique información no examinada o incluso maliciosa. El $51 \%$ de los expertos cree que la situación no mejorará en los próximos años y que en 2022 la mitad de las noticias serán fake news (Herrero-Diz et al., 2019).

Tandoc, Lim y Ling (2017) analizaron treinta y cuatro artículos académicos que utilizaron el término 'fake news' entre 2003 y 2017 y establecieron una taxonomía para clasificar este tipo de artículos en base a niveles de facticidad y engaño: sátira noticiosa, parodia noticiosa, fabricación, manipulación, publicidad y propaganda. Habgood-Coote (2018), sin embargo, aboga por que los periodistas y académicos dejen de usar los términos "fake news" y "posverdad", argumentando que tienen connotaciones propagandísticas que refuerzan las prácticas antidemocráticas. Hay evidencia de diferentes perspectivas que se refieren al mismo concepto. Aun así, el desorden de la información (Wardle y Derekhshan, 2017) que estamos presenciando actualmente es algo sin precedentes. Además, cada vez es más difícil distinguir entre la información sin sentido que pueden crear los algoritmos y la que se fabrica con intención, generalmente para ganar dinero (Bridle, 2018), así como el uso de noticias dirigidas, personal y emocionalmente producidas por el periodismo algorítmico (Bakir y McStay, 2017). Es aún más sorprendente cuando la desinformación se genera como resultado de accidentes, tragedias o crisis de salud, como fue el caso de los mensajes engañosos difundidos después del accidente del vuelo MH17 de Malaysia Airlines en el este de Ucrania (Rietjens, 2017) o durante la pandemia de COVID-19 (Frenkel, Alba y Zhong, 2020). 
Sixto-García, J., Rodríguez-Vázquez, A-I., \& López-García, X. Sistemas de verificación en medios nativos digitales e implicación de la audiencia en la lucha contra la desinformación en el modelo ibérico.

Independientemente del nombre que se le dé a las 'noticias falsas', numerosos proyectos de investigación, políticos y académicos han intentado, en los últimos años, valorar el papel de la alfabetización digital en el fortalecimiento de la resiliencia de los ciudadanos contra la desinformación, especialmente a partir de la escalada de crisis percibidas después de las elecciones y los resultados del referéndum en los EE.UU. y el Reino Unido, respectivamente (McDougall et al., 2019). La búsqueda de un antídoto contra la desinformación es una preocupación constante para organismos e instituciones supranacionales como la UNESCO (Ireton y Posetti, 2018) o la Comisión Europea $(2020,2018)$. El plan de acción de la Comisión Europea (2018) para intensificar los esfuerzos para contrarrestar la desinformación en el mundo se basa en cuatro aspectos clave: 1) mejor detección, análisis y exposición de la desinformación; (2) mayor cooperación y respuestas conjuntas a las amenazas; (3) mejor colaboración con plataformas en línea y la industria de los medios de comunicación; y (4) sensibilizar y mejorar la resiliencia social.

El proyecto DIGCOMP (Comisión Europea), el modelo Krumsvik (Noruega), el modelo TPACK (EE.UU.), el modelo JISC (Reino Unido), los estándares ISTE (EE.UU.) y el modelo P21 (EE.UU.) son ejemplos de modelos de desarrollo de alfabetización digital, destacando la necesidad emergente de políticas educativas (Pérez-Escoda, García-Ruiz y Aguaded, 2019, Brites, 2017). Los bibliotecarios (Frederiksen, 2017) también han advertido que discernir entre información falsa y real se está volviendo cada vez más complejo, argumentando que la única solución posible a esto radica en la alfabetización digital.

Hay otras voces críticas como Buckingham (2019), que advierte que limitar la solución a las fake news a la alfabetización digital es un enfoque reduccionista que no resolvería el problema. El autor promueve un concepto más amplio de alfabetización mediática, basado no solo en la tecnología, sino también en el pensamiento crítico sobre las dimensiones económicas, ideológicas y culturales de los medios. Dicho esto, ha reconocido la magnitud del problema y ha subrayado las dificultades que entraña su superación. Gutiérrez-Martín, Torrego-González y Vicente-Mariño (2019) señalan la necesidad de reivindicar la verdad como valor periodístico y social en el contexto digital, y que la alfabetización mediática se incluya como tarea imprescindible de todos los agentes educativos, es decir, la escuela, medios de comunicación y grupos sociales.

Egelhofer y Lecheler (2018) entienden que las fake news representan un cambio sin precedentes en la opinión pública y política sobre lo que el periodismo representa y cómo se construye el proceso de producción de información en un mundo digitalizado. Argumentan que las noticias falsas constituyen un fenómeno de comunicación bidimensional; que, en primer lugar, esta etiqueta se refiere a la práctica de la creación deliberada de desinformación pseudo-periodística y, en segundo lugar, que el término se usa en intentos de deslegitimar a los medios a través de la instrumentalización política.

Para que una información sea una fake news, obviamente debe ser falsa y estar creada con la intención de engañar. Además, también debe presentarse en un formato periodístico que imite el contenido de los medios (Lazer et al., 2017; Horne y 
Sixto-García, J., Rodríguez-Vázquez, A-I., \& López-García, X. Sistemas de verificación en medios nativos digitales e implicación de la audiencia en la lucha contra la desinformación en el modelo ibérico.

Adali, 2017). Son distintos los engaños o rumores difundidos por particulares, pero sin la apariencia de un producto periodístico, por ejemplo, a través de mensajes de audio de WhatsApp. En este caso, los destinatarios no interpretan este contenido como periodístico porque carece del formato intrínseco a ese tipo de producto, aunque la intención de los autores es igualmente despreciable. Varias investigaciones previas (Guess, Nyhan y Reifler, 2018; Lazer et al., 2018; Nelson y Taneja, 2018; Howard et al., 2017; Silverman, 2016) concluyeron que las fake news se difunden principalmente a través de las redes sociales gracias a la viralidad que ganan en esos espacios (Allcott, Gentzkow y Yu, 2019), algo no permitido dentro de los medios tradicionales (Carlson, 2016). Mientras tanto, aquellas noticias que son inexactas, pero que no ocultan un interés deliberado en engañar al público, no pueden considerarse fake news. Se denominan simplemente "noticias erróneas" (Egelhofer y Lecheler, 2018).

\subsection{Sistemas de verificación en los medios}

El fenómeno de las fake news no solo afecta a los ciudadanos, también a las empresas periodísticas que se enfrentan a diario a procesos de verificación de información cada vez más exigentes. Por un lado, deben verificar qué información viral o publicada en otros medios es realmente cierta. Por otro, deben garantizar la confiabilidad de los datos y la información que es enviada por el público a través de los mecanismos de participación ciudadana (Malmelin y Villi; 2016, Napoli, 2010) y de los espacios de crecimiento diseñados para la co-creación (Frow et al., 2015; Aitamurto, 2013; Ind y Coates, 2013).

Es necesario saber cómo los medios de comunicación afrontan estos desafíos. Esto es especialmente cierto en el contexto moderno, donde la credibilidad y legitimidad de los medios son cada vez más cuestionadas (Carlson, 2018) ya que también enfrentan una fuerte competencia por la democratización de la cultura (MorenoCaballud, 2014). Estudios previos han concluido que la verificación de noticias se lleva a cabo solo después de que se ha difundido la información errónea (Shao et al., 2018). Además, los estudios han encontrado que las advertencias de verificación de hechos difundidas por los operadores de redes sociales apenas fueron efectivas (Pennycook et al., 2020; Allcott y Gentzkow, 2017). Facebook, por ejemplo, terminó retirándolos ya que este tipo de etiquetado resultó ser completamente contraproducente y solo condujo a la exhibición de engaños (Lyons, 2017). La estrategia actual de Facebook se basa en reducir la distribución de este tipo de contenido entre los usuarios, aunque no lo elimina. La suposición de que una noticia es verdadera, simplemente porque no fue etiquetada como falsa, fue muy problemática. No obstante, una investigación reciente confirmó que los usuarios ahora son más escépticos sobre el contenido que consumen en las redes (Clayton et al., 2019).

Un estudio actual presenta la efectividad de interfaces web como BaitWatcher en la detección de titulares de noticias incongruentes, concluyendo que es necesario desarrollar una herramienta interpretable de inteligencia artificial (IA) para el diseño de una mejor interfaz con el fin de mitigar los efectos de la desinformación en línea (Park et al., 2020). Recientemente, han aparecido otras soluciones relacionadas con 
Sixto-García, J., Rodríguez-Vázquez, A-I., \& López-García, X. Sistemas de verificación en medios nativos digitales e implicación de la audiencia en la lucha contra la desinformación en el modelo ibérico.

la inteligencia artificial y la automatización de contenido, como Google Fact Check Tools. Este tipo de iniciativas están en la misma línea de las recomendaciones de Pomerleau y Rao (2016) en cuanto a la creación de una base de datos sobre noticias falsas. No obstante, su eficacia aún no se ha demostrado. En WhatsApp, por ejemplo, el cifrado de mensajes no permite que la inteligencia artificial examine su contenido. Por este motivo, la empresa decidió limitar los mensajes reenviados durante la pandemia de coronavirus como una forma de combatir la desinformación (Davis, 2020).

Debemos destacar el papel que juegan organizaciones como el Fake News Challenge, -integrado por más de 100 voluntarios y 71 equipos de la industria de la academia y el periodismo de todo el mundo-, para impulsar el desarrollo de herramientas de verificación basadas en inteligencia artificial, aprendizaje automático y procesamiento del lenguaje natural. Creen que "las tecnologías de IA son prometedoras para automatizar significativamente parte del proceso que los encargados de verificar los hechos utilizan hoy en día para determinar si una historia es real o un engaño" (Fake News Challenge, 2020). First Draft es otra iniciativa interesante formada por organizaciones reunidas por el Google News Lab, entre ellas Facebook o Twitter.

Otras organizaciones y asociaciones apuestan firmemente por el valor humano como herramienta fundamental para la verificación de los hechos. Un ejemplo relevante es la International Fact Checking Network (IFCN), una unidad del Instituto Poynter creada en 2015 y que cuenta con 67 medios asociados de todo el mundo. El Código de Principios (2016) que deben cumplir los medios de comunicación de esta unidad consta de cinco puntos: (1) compromiso de no partidismo y equidad; (2) compromiso con la transparencia de las fuentes; (3) compromiso con la transparencia en el financiamiento y la organización; (4) compromiso con la transparencia de la metodología; y (5) compromiso con correcciones abiertas y honestas. En 2017, Facebook anunció que registrarse en este código era un requisito previo para sus verificadores de datos.

The Trust Project es un consorcio internacional de medios creado en 2015 y con sede en el Markkula Center for Applied Ethics en la Universidad de Santa Clara. Entre sus más de 70 miembros se encuentran periódicos como The Washington Post y The Independent. Siguen criterios muy similares a los de IFCN en la lucha contra la desinformación y la verificación de hechos y noticias. NewsGuard califica el nivel de confiabilidad de los medios en una escala del 1 al 100 de acuerdo con nueve criterios relacionados con la transparencia y la información rigurosa. Además, crearon una extensión para navegadores de Internet (Chrome, Safari, Firefox y Edge) que permite al usuario certificar si un sitio web es confiable o no. Este verificador se puso en marcha en 2018, y al año siguiente, 500 páginas ya habían mejorado su metodología de trabajo periodístico siguiendo los criterios marcados por esta herramienta (Mondéjar, 2019).

En España, Maldita.es es un sitio de herramientas periodísticas que detecta información falsa y maliciosa. Junto con Newtral, son las únicas organizaciones españolas que forman parte del Grupo de Alto Nivel (HLEG) sobre noticias falsas y 
desinformación, formado por la Comisión Europea en 2018. El proceso de verificación de datos que sigue se basa en tres pasos: (1) un miembro del equipo investiga la desinformación, se pone en contacto con las fuentes primarias, contrasta la información con la de las bases de datos oficiales y utiliza procesos tecnológicos para identificar imágenes o audios; (2) el resto del equipo plantea dudas sobre la verificación a través de un grupo en Telegram y (3) después de examinar el trabajo del verificador, todo el equipo vota sobre la réplica de la noticia. Si hay un solo voto en contra de la réplica, debe comenzar de nuevo todo el proceso desde el principio. Todos los miembros del equipo también tienen derecho a un veto, lo que significa que, si un solo miembro veta una réplica, esta ya no se publicará. Newtral es un emprendimiento independiente cuya única accionista es la periodista española Ana Pastor. Es directora y presentadora de 'El Objetivo', la primera iniciativa de verificación de hechos en España, y de la que emanó Maldita.es. En 2019, después de que se rompiera su contrato con Associated Press y el sitio web de verificación norteamericano Snopes, Facebook firmó Maldita.es y Newtral para combatir las fake news (Pérez, 2019). Al hacerlo, Facebook los unió a France Presse como parte de su esfuerzo por disminuir el flujo de desinformación en todo el mundo.

En resumen, todas las iniciativas mencionadas intentan utilizar el potencial humano dentro de sus procesos de verificación de hechos, sin arriesgar el papel complementario y de apoyo que los sistemas de IA pueden desempeñar dentro de estas tareas. Pero, ¿qué pasa con los propios medios de comunicación? La Comisión Europea (2018) aconseja tanto a las organizaciones de prensa escrita como a las emisoras que intensifiquen sus esfuerzos para lograr los más altos niveles de cumplimiento de los estándares éticos y profesionales con el objetivo de mantener un ecosistema de medios confiable y pluralista. Valoramos la existencia de órganos colegiados, asociaciones y entidades que luchan contra el grave problema de la desinformación. Sin embargo, estos esfuerzos deben traducirse en mecanismos de trabajo y rutinas productivas en las redacciones. Es prácticamente imposible investigar la intención detrás de la producción de noticias falsas. No obstante, se entiende que es importante brindar a la comunidad científica y académica casos de cómo los medios de comunicación combaten la desinformación entre bastidores, y que conozcan qué sistemas se utilizan para capturar intenciones maliciosas. En línea con los aportes de Hoxha y Hanitzsch (2018), también se argumenta aquí que entrevistar a los periodistas es fundamental para identificar los procesos que utilizan para crear noticias en el contexto actual.

\section{OBJETIVOS}

Esta investigación tiene dos objetivos: (1) demostrar la existencia de sistemas de verificación en medios digitales nativos y (2) analizar el papel de la audiencia en la lucha contra la desinformación. Este trabajo tiene un gran interés en los medios digitales nativos, donde se crean contenidos en y para Internet, ya que es este formato el que brinda a la audiencia mayores posibilidades de participación y cocreación. Se tomó la decisión de estudiar el modelo ibérico, por lo que se seleccionaron los principales medios nativos digitales de España y Portugal. Las selecciones se basaron en el porcentaje de uso semanal más alto según los datos 
Sixto-García, J., Rodríguez-Vázquez, A-I., \& López-García, X. Sistemas de verificación en medios nativos digitales e implicación de la audiencia en la lucha contra la desinformación en el modelo ibérico.

recopilados en Digital News Report 2019 (Reuters Institute, 2019). La selección española fue "eldiario.es" y la portuguesa "Observador".

La selección se justifica por los datos de audiencia y el conocimiento de marca de los periódicos y, por tanto, se trata de una muestra no probabilística que se utiliza en escenarios donde la población es variable y pequeña. Por tanto, resulta oportuno (Igartua, 2006) seleccionar casos que sean característicos de ese universo (LópezRoldán y Fachelli, 2015; Otzen y Manterola, 2017). Los resultados se presentan desde una perspectiva comparativa, ya que la técnica de los estudios comparativos tiene una larga tradición y es muy utilizada en diferentes áreas del conocimiento, desde la medicina (Hietanen et al., 2005) hasta la ciencia veterinaria (Calvo, Abarca y Trape, 1981) a los estudios filológicos (Guijarro-Fuentes, 2005).

\section{MATERIAL Y MÉTODOS}

De acuerdo con las líneas de investigación propuestas por Hoxha y Hanitzsch (2018), se realizaron entrevistas a los responsables de gestionar el objeto de estudio en los medios analizados (editores de audiencia, editores de engagement o editores de analítica). Para ello, se desarrolló un cuestionario que combina preguntas de carácter cuantitativo y cualitativo, atendiendo a los objetivos secundarios de la investigación.

1. Identificar quién se encarga de gestionar las relaciones con las audiencias en los medios digitales nativos, recogiendo la denominación del perfil profesional (editor de audiencia, editor de engagement, editor de crecimiento, analista de impacto y métricas, editor multiplataforma, editor de Lab Media, editor de Social Media...) y comprobar si forma parte de un departamento unipersonal o multipersonal.

2. Examinar las evaluaciones de los responsables de los medios de comunicación sobre los sistemas de participación ciudadana y los mecanismos de cocreación habilitados para los públicos.

3. Verificar la existencia de sistemas de verificación de noticias en los medios nativos digitales.

4. Analizar la participación de las audiencias en los procesos de verificación.

De acuerdo con los objetivos de la investigación, el cuestionario se estructuró en cinco bloques temáticos. Los bloques 1,3 y 4 están relacionados con la recolección de información cuantitativa, mientras que los bloques 2,5 y 6 están orientados a la recolección de información cualitativa:

1. Identificación del perfil profesional empleado en la redacción y ubicación de perfiles específicos para trabajar con audiencias (editor de audiencia, editor de engagement, editor de crecimiento, editor de impacto y métricas, editor multiplataforma, editor de Lab Media, editor de Social Media u otros), así 
Sixto-García, J., Rodríguez-Vázquez, A-I., \& López-García, X. Sistemas de verificación en medios nativos digitales e implicación de la audiencia en la lucha contra la desinformación en el modelo ibérico.

como una descripción del organigrama de la empresa y estructuración de los recursos humanos asignados al departamento.

2. Análisis de sinergias y relaciones entre el departamento de relaciones con la audiencia y la unidad organizativa.

3. Localización de estrategias de verificación de contenidos, asignación a áreas o secciones en las redacciones y herramientas de verificación de datos utilizadas.

4. Implicación de la audiencia en los procesos de verificación de datos a través del registro de usuarios, personalización, calificación de noticias, comentarios, encuestas, blog de lectores, comunidades de usuarios más cerradas (diálogo privado entre los medios y la audiencia), comunidades de usuarios cerradas para temas específicos (o secciones temáticas), chatbots, contenido generado por el usuario (CGU) u otros, así como análisis de métodos de medición de resultados como Google Trends, Trendsmap u otros.

5. Presentación de ejemplos de participación de la audiencia en otros medios y exploración de alternativas similares. Específicamente, se ofrecieron los siguientes ejemplos: (a) Grupo PostThis en el Facebook de The Washington Post, un grupo moderado y cerrado para lectores interesados en historias de periodismo de rendición de cuentas; (b) El Centro de Lectores de The New York Times para construir vínculos más profundos con la audiencia y desde donde los periodistas hablan directamente con los lectores sobre la cobertura de noticias; (c) News and Brews de NPR de Illinois (EE.UU.) para fomentar los encuentros cara a cara con el público.

6. Desarrollo de iniciativas de co-creación.

Se plantearon un total de 23 preguntas. El cuestionario incluía una mezcla de preguntas cerradas (dicotómicas) y abiertas, según el caso. Al final del cuestionario, se establecieron una serie de ítems para una evaluación gradual. Para esta última parte se utilizó como referencia la escala de calificación Likert (Wigley, 2013), aunque se adaptó a una escala básica de tres opciones (en desacuerdo, de acuerdo, totalmente de acuerdo) para facilitar el trabajo de respuesta de los periodistas e identificar tendencias. El tiempo medio de respuesta fue de 27,5 minutos.

El contacto con los medios de comunicación se estableció el 26 de febrero de 2020 por correo electrónico y teléfono. De eldiario.es se entrevistó a Ander Oliden, jefe de Información y editor en jefe de portada y redes sociales. De Observador, se entrevistó a Raquel Rosa, la encargada de suscripciones.

\section{DISCUSIONES}

En primer lugar, en "eldiario.es" existe un departamento unipersonal encargado de gestionar y mantener el contacto con las audiencias. El título de la persona a cargo es "director de nuevas audiencias" y cuenta con el apoyo de los editores de 
Sixto-García, J., Rodríguez-Vázquez, A-I., \& López-García, X. Sistemas de verificación en medios nativos digitales e implicación de la audiencia en la lucha contra la desinformación en el modelo ibérico.

redes sociales del equipo de portada y redes. Ambos conjuntos de perfiles profesionales dependen en gran medida de sus habilidades de escritura. En "Observador", en cambio, esta función está encomendada al Departamento de Social Media, integrado por seis personas que trabajan en turnos rotativos, y que también dependen de la Sala de Redacción.

Eldiario.es afirma utilizar herramientas de verificación para corroborar la información que publica. "Todo el equipo de redacción trabaja en tareas de verificación y tenemos un convenio de colaboración con Maldita.es para el desarrollo de una sección (El Detector) y una colaboración semanal para Desalambre, nuestra sección de Derechos Humanos" (A. Oliden, comunicación personal, marzo 5, 2020). Sin embargo, reconocen que no involucran a las audiencias en la verificación de la información. El Observador no brindó mucha información sobre este tema, alegando "utilizarla" (R. Rosa, comunicación personal, 19 de marzo de 2020), aunque también descuidando la participación de la audiencia.

"Eldiario.es" ofrece un abanico bastante amplio de mecanismos de participación ciudadana (comentarios, encuestas, blog de lectores, etc.). También brindan oportunidades para la creación conjunta de contenido (la capacidad de enviar filtraciones confidenciales, corregir textos [solo socios], comentarios tomados en cuenta por la sala de redacción [solo miembros], votación en línea [solo miembros] y encuentros cara a cara entre periodistas y suscriptores). Esto significa que la información del público podría terminar en algún lugar dentro de la producción escrita de "eldiario.es", o al menos podría aparecer como parte de una publicación web. En "Observador", las oportunidades tanto de participación ciudadana (registro de usuarios, comentarios o personalización de la app) como de co-creación (corrección de textos y envío de contenidos generados por los usuarios) son más limitadas. Rosa afirmó "permitimos comentarios sobre nuestros artículos. También permitimos que los usuarios se registren para suscribirse a boletines y tener acceso a nuestro contenido Premium. También nos relacionamos con nuestra audiencia a través de Facebook Messenger cada vez que una persona se pone en contacto con nosotros" (comunicación personal, 19 de marzo de 2020).

El redactor en jefe de eldiario.es entiende que estos mecanismos "constituyen una forma de participación de la audiencia en el sentido de que son un medio de contacto con la redacción" (A. Oliden, comunicación personal, 5 de marzo de 2020). Además, aclaró que "a veces, cuando el comentario se refiere a temas relacionados con el contenido, los periodistas se comunican con los lectores para discutir el tema". Oliden también explica que "reciben periódicamente a miembros de eldiario.es en nuestra sala de redacción para conversar con el director, los periodistas y otros miembros del equipo del diario. Suele haber discusión de cobertura, cambios previstos y se responden preguntas sobre el funcionamiento de la redacción. También es útil buscar la opinión de la comunidad de socios sobre nuestro contenido" (comunicación personal, 5 de marzo de 2020). Rosa dice que en el diario portugués "han concertado iniciativas de contacto directo" y que "han organizado algunas conferencias presenciales", que consideran que son "una forma positiva de relacionarse con nuestra audiencia" (comunicación personal, 19 de marzo de 2020). 
Sixto-García, J., Rodríguez-Vázquez, A-I., \& López-García, X. Sistemas de verificación en medios nativos digitales e implicación de la audiencia en la lucha contra la desinformación en el modelo ibérico.

En cuanto a la co-creación de contenidos, Oliden confirma que "han puesto en marcha algunas iniciativas para solicitar la participación en diferentes temas con los que desarrollar historias a partir de la experiencia de nuestros lectores", pero que, no obstante, "se siguen preparando iniciativas como manera de mejorar esos flujos de participación" (comunicación personal, 5 de marzo de 2020). En Observador, el gerente de suscripción manifestó que alientan a su audiencia "a enviarnos correos electrónicos siempre que tengan una corrección o sugerencia a nuestros artículos", pero que en la actualidad "no están desarrollando nuevos enfoques" (R. Rosa, comunicación personal, 19 de marzo de 2020).

Ambos medios analizados muestran un gran interés en incorporar audiencias a las redacciones e involucrarlas en la labor periodística. Con algunas diferencias en la graduación (ver Tabla 1), existe una tendencia obvia a promover el contacto con el público. Esto también es evidente en el uso de espacios fuera de línea, ya que los responsables de los medios entienden que aumentar la participación de la audiencia dentro de sus medios también aumenta la calidad de la información. Ambos medios son fuentes comprometidas con el establecimiento de espacios de trabajo directos entre editores y público y valoran positivamente las experiencias previas que han desarrollado. Desde el punto de vista de los espacios destinados a la co-creación, las respuestas son congruentes y confirman que los medios de comunicación tienen interés en mejorar los sistemas de participación con las audiencias y en habilitar nuevos espacios para que los públicos puedan co-crear sus propios productos.

"Eldiario.es" y "Observador" manifiestan igualmente su voluntad de involucrar al público en la producción de información. Descartan por completo limitar su medición del impacto de las noticias en la audiencia utilizando únicamente métricas y analíticas proporcionadas por algoritmos de sitios web, aplicaciones o redes sociales. Sin embargo, ninguna de las fuentes de los medios sostiene que el público debería participar en la verificación de los hechos; limitan esta tarea solo a los periodistas. 
Sixto-García, J., Rodríguez-Vázquez, A-I., \& López-García, X. Sistemas de verificación en medios nativos digitales e implicación de la audiencia en la lucha contra la desinformación en el modelo ibérico.

Tabla 1. Evaluación de la participación de la audiencia en los medios

\begin{tabular}{|c|c|c|c|c|c|c|c|}
\hline \multirow[b]{2}{*}{ Elementos a evaluar } & \multicolumn{2}{|c|}{$\begin{array}{l}\text { Totalmente } \\
\text { en } \\
\text { desacuerdo }\end{array}$} & \multicolumn{2}{|c|}{$\begin{array}{l}\text { De } \\
\text { acuerdo }\end{array}$} & \multicolumn{2}{|c|}{$\begin{array}{l}\text { Totalmente } \\
\text { de acuerdo }\end{array}$} & No lo sé \\
\hline & ES & PT & ES & PT & ES & PT & ES PT \\
\hline $\begin{array}{l}\text { El diálogo directo con la audiencia en el espacio } \\
\text { online es fundamental para los medios. }\end{array}$ & & & & $x$ & $x$ & & \\
\hline $\begin{array}{l}\text { El encuentro directo entre los editores y el } \\
\text { público en el entorno offline (cafés, reuniones...) } \\
\text { es fundamental. }\end{array}$ & & & & $X$ & $X$ & & \\
\hline $\begin{array}{l}\text { La creación de comunidades cerradas en los } \\
\text { medios, involucrando a la audiencia en el } \\
\text { trabajo periodístico, ayuda a mejorar la calidad } \\
\text { de la información. }\end{array}$ & & & $X$ & & & $X$ & \\
\hline $\begin{array}{l}\text { Soy partidario de abrir espacios de trabajo } \\
\text { directos entre editores y público. }\end{array}$ & & & $X$ & $X$ & & & \\
\hline $\begin{array}{l}\text { He tenido experiencias similares con la audiencia } \\
\text { y me parecen muy positivas. Me gusta trabajar } \\
\text { en contacto directo con la audiencia. }\end{array}$ & & & $X$ & $X$ & & & \\
\hline $\begin{array}{l}\text { Creo que el diario para el que trabajo tiene } \\
\text { interés en mejorar la participación de la } \\
\text { audiencia y abrir nuevos espacios de co- } \\
\text { creación. }\end{array}$ & & & $x$ & $x$ & & & \\
\hline $\begin{array}{l}\text { El periódico para el que trabajo solo mide el } \\
\text { impacto de las noticias en la audiencia con } \\
\text { métricas de consumo en la web, aplicaciones y } \\
\text { redes sociales, pero no tiene interés en } \\
\text { involucrar a la audiencia en la producción de } \\
\text { información. }\end{array}$ & $x$ & $x$ & & & & & \\
\hline $\begin{array}{l}\text { Creo que los medios de comunicación deben } \\
\text { involucrar al público en la verificación de la } \\
\text { noticia. }\end{array}$ & $X$ & $X$ & & & & & \\
\hline
\end{tabular}

$$
\begin{array}{ll}
\hline \text { ES } & \text { España } \\
\hline \text { PT } & \text { Portugal } \\
\hline
\end{array}
$$

Fuente: Elaboración propia

\section{CONCLUSIONES-RESULTADOS DE INVESTIGACIÓN}

Según el objetivo 1, los medios de comunicación analizados en este estudio valoran mucho la participación de la audiencia. Por ello, han habilitado espacios tanto de participación ciudadana como de co-creación. Aunque las mayores oportunidades de implicación se encuentran en los medios digitales, estos espacios también requieren perfiles profesionales especializados en la gestión de estas relaciones. En "eldiario.es", estas responsabilidades de gestión las realiza un departamento unipersonal (director de nuevas audiencias), mientras que en "Observador" lo realiza un equipo de seis personas (departamento de Social Media). Ambos periódicos entienden que la participación de las audiencias mejora la calidad del trabajo periodístico. En consecuencia, han desarrollado modelos de participación 
Sixto-García, J., Rodríguez-Vázquez, A-I., \& López-García, X. Sistemas de verificación en medios nativos digitales e implicación de la audiencia en la lucha contra la desinformación en el modelo ibérico.

en la web y en las redes sociales, y también encuentros presenciales entre audiencias y periodistas.

En segundo lugar, y quizás sorprendentemente, a pesar de la importancia que se le da al público; ni "eldiario.es" ni "Observador" pretenden, ni consideran necesario, integrar a sus audiencias en sus procesos de verificación de noticias (objetivo 2). Más bien, sostienen que este trabajo debe limitarse a los periodistas profesionales. En ningún caso recurren a la ciudadanía para contrastar hechos o verificar noticias, aunque esto podría ser útil en situaciones en las que miembros del público general son fuentes implicadas o testigos presenciales de los hechos. Tampoco se verifica la existencia de mecanismos de inteligencia artificial. Lo que es evidente es un fuerte compromiso con el talento humano como lo demuestra la adhesión de "eldiario.es" a "Maldita.es".

Los autores coinciden con la noción de los dos medios de comunicación aquí planteados, de que ellos mismos son los responsables en última instancia de las noticias que publican. No obstante, este trabajo también aboga por la asignación de más recursos y más acción humana en la verificación de los contenidos generados por los usuarios. A modo de ejemplo, según datos de ComScore (2020), durante el brote de COVID-19, los medios tradicionales han recuperado poder y sus publicaciones están siendo vistas con más credibilidad que las redes sociales. A medida que los espacios para la co-creación y el periodismo ciudadano se vuelven más frecuentes, también lo hace la necesidad de capacitar a las audiencias en habilidades mediáticas y digitales. Hasta que esto se logre, los medios necesitan integrar más perfiles profesionales dedicados a la verificación y filtrar en el tiempo y forma lo que se publica en los espacios dedicados al periodismo ciudadano. No hacerlo podría significar que los medios, especialmente los medios digitales, terminen albergando no solo información falsa, sino también fake news. Incluso bajo el supuesto utópico de una sociedad plenamente competente en competencias digitales y mediáticas, siempre existirá la posibilidad de que ciudadanos malintencionados decidan engañar al resto si se ignora el trabajo del periodista.

La tabla 2 muestra las conclusiones con respecto a los objetivos secundarios de la investigación. Identifica los perfiles profesionales encargados de gestionar las relaciones con la audiencia en cada uno de los medios, los mecanismos de implicación de la audiencia, el grado de implantación de los sistemas de co-creación y la implicación de los lectores en la verificación de la noticia. 
Sixto-García, J., Rodríguez-Vázquez, A-I., \& López-García, X. Sistemas de verificación en medios nativos digitales e implicación de la audiencia en la lucha contra la desinformación en el modelo ibérico.

Tabla 2. Audiencia y verificación de hechos en cada medio

\begin{tabular}{|c|c|c|}
\hline & Eldiario.es & Observador \\
\hline $\begin{array}{l}\text { Perfil profesional específico } \\
\text { para trabajar con público. }\end{array}$ & $\mathrm{Si}$ & Si \\
\hline $\begin{array}{l}\text { Identificación del perfil } \\
\text { profesional de relaciones } \\
\text { con la audiencia }\end{array}$ & $\begin{array}{ll}\text { - } & \text { Director de } \\
\text { nuevas } \\
\text { audiencias } \\
\text { - Editores de redes } \\
\text { sociales en la página } \\
\text { principal y en el } \\
\text { equipo de la red }\end{array}$ & Departamento de Redes Sociales \\
\hline $\begin{array}{l}\text { Recursos humanos para las } \\
\text { relaciones con la audiencia }\end{array}$ & Equipo & $\begin{array}{c}\text { Equipo de } 6 \text { personas con turnos } \\
\text { rotativos }\end{array}$ \\
\hline $\begin{array}{l}\text { Área de trabajo y } \\
\text { situaciones de } \\
\text { organización }\end{array}$ & Oficina de redacción & Oficina de redacción \\
\hline $\begin{array}{l}\text { Uso de herramientas de } \\
\text { verificación }\end{array}$ & Si & Si \\
\hline $\begin{array}{l}\text { Recursos humanos para la } \\
\text { verificación de datos }\end{array}$ & $\begin{array}{l}\text { Equipo editorial } \\
\text { - } \quad \text { Acuerdo de colaboración } \\
\text { con Maldita.es } \\
\text { (plataforma de } \\
\text { verificación de datos) } \\
\text { para una sección (El } \\
\text { Detector) y una sección } \\
\text { semanal para } \\
\text { Desalambre, la sección } \\
\text { de Derechos Humanos }\end{array}$ & Equipo editorial \\
\hline $\begin{array}{l}\text { Participación de la audiencia } \\
\text { en la verificación de datos }\end{array}$ & No & No \\
\hline $\begin{array}{l}\text { Vías de participación y co- } \\
\text { creación con audiencias }\end{array}$ & $\begin{array}{ll}\text { - } & \text { Registro de usuario } \\
\text { - } & \text { Personalización } \\
\text { - } & \text { Encuentarios } \\
\text { - } & \text { Blog de lectores } \\
\text { - } & \text { Espacios exclusivos } \\
& \text { para socios (reuniones } \\
& \text { con periodistas, } \\
& \text { eventos sobre el } \\
& \text { funcionamiento de los } \\
& \text { medios de } \\
\text { comunicación) } \\
\text { Charlas con el } \\
\text { director, periodistas y } \\
\text { otros miembros del } \\
\text { equipo. } \\
\text { eldiario.es } \\
\text { responde(espacio } \\
\text { para dudas o } \\
\text { quejas) } \\
\text { Conversiones con } \\
\text { socios. }\end{array}$ & $\begin{array}{l}\text { - Registro de usuario } \\
\text { - Comentarios } \\
\text { - Permitir que el usuario se } \\
\text { registre para suscribirse al boletín } \\
\text { y al contenido Premium. } \\
\text { - Interacción con la audiencia a } \\
\text { través de Facebook Messenger } \\
\text { siempre que una persona los } \\
\text { contacte. } \\
\text { - Conferencias presenciales. } \\
\text { - Iniciativas de contacto directo }\end{array}$ \\
\hline $\begin{array}{l}\text { Sistemas de medición } \\
\text { analítica }\end{array}$ & - & $\begin{array}{ll}\text { - } & \text { Chartbeat } \\
\text { - } & \text { Google Analytics. }\end{array}$ \\
\hline
\end{tabular}


Sixto-García, J., Rodríguez-Vázquez, A-I., \& López-García, X. Sistemas de verificación en medios nativos digitales e implicación de la audiencia en la lucha contra la desinformación en el modelo ibérico.

\begin{tabular}{lll}
$\begin{array}{l}\text { Comunicación mediática } \\
\text { con audiencias }\end{array}$ & $\begin{array}{l}\text { Cuando el comentario } \\
\text { trata sobre cuestiones } \\
\text { de contenido, los } \\
\text { editores se comunican } \\
\text { con los lectores. } \\
\text { Debate sobre la } \\
\text { cobertura de noticias } \\
\text { en reuniones } \\
\text { presenciales }\end{array}$ & $\begin{array}{l}\text { Animan a la audiencia a enviar } \\
\text { correos electrónicos siempre } \\
\text { que tengan una corrección o } \\
\text { una sugerencia }\end{array}$ \\
\hline Desarrollo de la co-creación & $\begin{array}{l}\text { Iniciativas para solicitar historias } \\
\text { de las experiencias personales } \\
\text { de los lectores. Están } \\
\text { preparando iniciativas para } \\
\text { mejorar los flujos de } \\
\text { participación }\end{array}$ \\
\hline
\end{tabular}

Fuente: Elaboración propia

Los medios de comunicación dan cada vez más importancia a la participación de la audiencia. Así se ha demostrado en escritos anteriores sobre las fórmulas de participación ciudadana (Malmelin y Villi, 2016; Napoli, 2010), sobre la provisión de espacios para la co-creación de contenidos en los que los usuarios puedan desarrollar su propia experiencia (Frow et al. , 2015; Aitamurto, 2013; Ind y Coates, 2013). Esta investigación ha verificado esos mismos hallazgos.

La implicación de las audiencias en los procesos relacionados con la producción de información en general, y en la elaboración de noticias en particular, parece muy positiva. Sin embargo, por otro lado, esta participación también constituye un riesgo importante si el contenido que brindan no se filtra adecuadamente a través de sistemas de verificación. La democratización de la cultura (Moreno-Caballud, 2014) trae grandes beneficios para la creatividad y para un acceso más equitativo a la información, pero se está dando en un contexto social, político, económico y tecnológico en el que el papel de los medios de comunicación parece cada vez más desacreditado y socavado (Carlson, 2018). Una de las causas más destacadas de esta situación se relaciona con la desinformación y la presencia de noticias falsas en los medios. Esto conduce a la pérdida de la confianza pública en los periodistas y erosiona gravemente el derecho a recibir información, lo que perjudica la calidad de la democracia dentro de los estados.

Para paliar esta situación, han surgido iniciativas en todo el mundo que apuestan por un periodismo de calidad, libre y pluralista. Los ejemplos incluyen los relacionados con la inteligencia artificial y el aprendizaje automático como las recientes Google Fact Check Tools, o los sistemas implementados por algunas redes sociales como Facebook. Medios y periodistas están cada vez más comprometidos con asociaciones, instituciones o movimientos como Fact Checking Network o The Trust Project que buscan agregar valor humano al proceso de detección de noticias falsas desarrollado por mecanismos y algoritmos automatizados.

Siguiendo las aportaciones de investigadores como Egelhofer y Lecheler (2018), este trabajo sostiene que es pertinente y necesario diferenciar entre las false news y 
fake news. El primero es una representación de una falta de contraste en las fuentes, incluso de una posible mala praxis en el ordenamiento de la información elaborada por los periodistas y publicada en los medios, mientras que el segundo se define por su intención de engañar. Por esta razón, las fake news se vuelven más frecuentes en épocas de gran convulsión social, donde el objetivo de su uso es desinformar. Ejemplos de esto son el Brexit y la pandemia causada por COVID-19. El poder potencial de las fake news aumenta cuando se presenta como periodismo profesional en diseño y apariencia (Lazer et al., 2017; Horne y Adali, 2017), dejando a muchos usuarios incapaces de identificar su falsedad.

Para paliar esta situación, las organizaciones supranacionales están apostando por la alfabetización digital o la alfabetización mediática como soluciones. Sin embargo, también hay una falta de consenso aquí. Hay quienes entienden que aumentar las habilidades en alfabetización digital resolvería este problema (Pérez-Escoda, GarcíaRuiz y Aguaded, 2019; Frederiksen, 2017). Pero también hay quienes ven esto como una visión reduccionista que debe complementarse con competencias en alfabetización mediática (Buckingham, 2019) y con la recuperación de la verdad como valor periodístico fundamental (Gutiérrez-Martín, Torrego-González y VicenteMariño, 2019).

Por su parte, los medios de comunicación tienen un gran desafío por delante. Además de seguir las recomendaciones éticas y deontológicas de las asociaciones profesionales nacionales e internacionales para la erradicación de la desinformación, este trabajo sostiene que los propios periodistas deben aplicar mecanismos de verificación en las redacciones. En el análisis del modelo ibérico de los medios nativos digitales más relevantes en España y Portugal, no se aprecian diferencias significativas entre los dos países. Más bien, se comparten tendencias y patrones de acción.

Si las fake news se infiltraran en los medios, sería prácticamente imposible revertir el daño causado a la reputación de los medios y del periodismo profesional. Los ciudadanos podrían perder la fe en la fiabilidad y credibilidad de los medios de comunicación, frente a las opiniones personales u otros sitios de Internet poco fiables. Una situación en la que los ciudadanos ven comprometido su derecho a recibir información veraz y verificada, seguramente significa una democracia frágil en general. Que el capital humano sea valorado, pero complementado con sistemas de inteligencia artificial, sería ideal dentro de estos procesos de verificación de datos. El papel de los periodistas sigue siendo esencial para verificar los hechos y combatir la desinformación en el mundo, ya que parece que la sociedad no puede depender únicamente de las máquinas para este tipo de trabajo.

\section{REFERENCIAS}

Aitamurto, T. (2013). Balancing Between Open and Closed: Co-creation in Magazine Journalism. Digital Journalism, 1(2), 229-251. https://doi.org/10.1080/21670811.2012.750150 
Sixto-García, J., Rodríguez-Vázquez, A-I., \& López-García, X. Sistemas de verificación en medios nativos digitales e implicación de la audiencia en la lucha contra la desinformación en el modelo ibérico.

Alcott, H., Gentzkow., M and Yu, C. (2019). Trends in the diffusion of misinformation on social media. Research and Politics, 1(8). https://doi.org/10.1177/2053168019848554

Allcott, H. and Gentzkow, M. (2017). Social media and fake news in the 2016 election. Journal of Economic Perspectives, 31(2), 211-36. https://doi.org/ 10.1257/jep.31.2.211

Bakir, V. and McStay, A. (2018). Fake News and The Economy of Emotions. Digital Journalism, 6(2), 154-175. https://doi.org/10.1080/21670811.2017.1345645

Bridle, J. (2018). New Dark Age: Technology and the End of the Future. Verso.

Brites, MJ. (Coord.) (2017). Digital literacy and education (2014-July 2016), national reports (Portugal, UK, Ireland, Spain, Serbia and Italy). ELN - European literacy network, digital literacy team (WG2). https://www.is1401eln.eu/en/gca/index.php?id=149

Buckingham, D. (2019). Teaching media in a 'post-truth' age: fake news, media bias and the challenge for media/digital literacy education. Culture and Education, 31(2), 213-231. https://doi.org/10.1080/11356405.2019.1603814

Calvo, MA., Abarca, L. and Trape, J. (1981). Comparative study Trichophyton Mentagrophytes and Trichophyton Verrucosum. Sabouraudia: Journal of Medical and Veterinary Mycology, https://doi.org/10.1080/00362178185380031

Carlson, M.(2018). The information politics of journalism in a post-truth age. Journalism Studies, 19(13), https://doi.org/10.1080/1461670X.2018.1494513

Carlson, M. (2016). Embedded links, embedded meanings: Social media commentary and news sharing as mundane media criticism. Journalism Studies, 17(7), 915924. https://doi.org/10.1080/1461670X.2016.1169210

Clayton, K., Blair, S., Busam, JA., Forstner, S., Glance, J., Green, G., Kawata, A., Kovvuri, A., Martin, J., Morgan, E., Sandhu, M., Sang, R., Scholz-Bright, R., Welch, A., Wolff, A., Zhou, A. and Nyhan, B. (2019). Real Solutions for Fake News? Measuring the Effectiveness of General Warnings and Fact-Check Tags in Reducing Belief in False Stories on Social Media. Polit Behav. https://doi.org/10.1007/s11109-019-09533-0

ComScore (2020). Traditional media regains power and credibility during the Coronavirus pandemic. https://bit.ly/2RIXplv

Davis, S. (April, 7, 2020). Could a limit on forwarding messages slow coronavirus fake news? Euronews. https://www.euronews.com/2020/04/07/could-a-limit-onforwarding-messages-slow-coronavirus-fake-news 
Sixto-García, J., Rodríguez-Vázquez, A-I., \& López-García, X. Sistemas de verificación en medios nativos digitales e implicación de la audiencia en la lucha contra la desinformación en el modelo ibérico.

Egelhofer, JL. and Lecheler, S. (2019). Fake news as a two-dimensional phenomenon: a framework and research agenda, Annals of the International Communication Association, 43(2), 97-116. https://doi.org/10.1080/23808985.2019.1602782

European Commission (2018). A multi-dimensional approach to disinformation: Report of the independent high level group on fake news and online disinformation. Publications Office of the European Union.

European Commission (2020). Fighting disinformation. https://ec.europa.eu/info/livework-travel-eu/health/coronavirus-response/fighting-disinformation en

Fact Checking Network (2016). Code of Principles. https://ifcncodeofprinciples.poynter.org/

Fake News Challenge (2020). Exploring how artificial intelligence technologies could be leveraged to combat fake news. http://www.fakenewschallenge.org/

Frederiksen, L. (2017). Fake News. Public Services Quarterly, 13(2), 103-107. https://doi.org/10.1080/15228959.2017.1301231

Frenkel, S., Alba, D. and Zhong, R. (March, 8, 2020). Surge of Virus Misinformation Stumps Facebook and Twitter. The New York Times. https://www.nytimes.com/2020/03/08/technology/coronavirus-misinformationsocial-media.html

Frow, P., Nenonen, S., Payne, A. and Storbacka, K. (2015) Managing co-creation design: A strategic approach to innovation. British Journal of Management, 26, 463-483. https://doi.org/10.1111/1467-8551.12087

Guess, A., Nyhan, B. and Reifler, J. (2018) Selective exposure to misinformation: Evidence from the consumption of fake news during the 2016 U. S. presidential campaign [Pdf File]. https://www.dartmouth.edu/ nyhan/fake-news-2016.pdf

Guijarro-Fuentes, P. (2005). Tiempo y Aspecto: estudio comparativo entre nativos y no nativos de nivel avanzado de español. Hispanic Research Journal, 6(2), 99-115. https://doi.org/10.1179/146827305X38760

Gutiérrez-Martín, A., Torrego-González, A. \& Vicente-Mariño, M. (2019). Media education with the monetization of YouTube: the loss of truth as an exchange value. Culture and Education, 31(2), 267-295. https://doi.org/10.1080/11356405.2019.1597443

Habgood-Coote, J. (2019). Stop talking about fake news! Inquiry, 62(9-10), 10331065. https://doi.org/10.1080/0020174X.2018.1508363

Herrero-Diz, P., Conde-Jiménez, J., Tapia-Frade, A. and Varona-Aramburu, D. (2019). The credibility of online news: an evaluation of the information by 
Sixto-García, J., Rodríguez-Vázquez, A-I., \& López-García, X. Sistemas de verificación en medios nativos digitales e implicación de la audiencia en la lucha contra la desinformación en el modelo ibérico.

university students. Culture and Education, 31(2), 407-435. https://doi.org/10.1080/11356405.2019.1601937

Hietanen, A., Era, P., Henrichsen, J., Rosenhall, U., Sorri, M. and Heikkinen, E. (2005). Hearing among 75-year-old people in three Nordic localities: A comparative study. International Journal of Audiology, 44(9), 500-508. https://doi.org/10.1080/14992020500189112

Horne, BD. and Adali, S. (2017). This just in: Fake news packs a lot in title, uses simpler, repetitive content in text body, more similar to satire than real news. arXiv:1703.09398 [cs.SI] [Pdf File]. https://arxiv.org/abs/1703.09398

Hoxha, A. and Hanitzsch, T. (2018). How conflict news comes into being: Reconstructing 'reality' through telling stories. Media, War \& Conflict, 11(1), 4664. https://doi.org/10.1177\%2F1750635217727313

Igartua, JJ. (2006) Métodos cuantitativos de investigación en Comunicación. Bosch.

Ind, N. and Coates, N. (2013). The meanings of co-creation. European Business Review, 25(1), 86-95.

Ireton, C. and Posett,i J. (Eds) (2018). Journalism, 'fake news' and disinformation: Handbook for journalism education and training. Unesco.

Lazer, D., Baum, M., Benkler, Y., Berinsky, A., Greenhill, K,. Menczer, F., Metzger, M., Nyhan, B., Pennycook, G., Rothschild, D., Schudson, M., Sloman, S., Sunstein, C., Thorson, E., Watts, D. and Zittrain, J. (2018). The science of fake news. Science 359(6380), 1094-1096. https://doi.org/10.1126/science.aao2998

Lazer, D., Baum, M., Grinberg, N., Friedland, L., Joseph, K., Hobbs, W. and Mattsson, C. (2017). Combating fake news: An agenda for Research and Action [Pdf Files]. https://shorensteincenter.org/wp-content/uploads/2017/05/Combating-FakeNews-Agenda-for-Research-1.pdf

López-Roldán, P. and Fachelli, S. (2015). El diseño de la muestra. In: P. LópezRoldán and S. Fachelli (eds.) Metodología de la investigación social cuantitativa. Universitat Autònoma de Barcelona.

Lyons, T. (December 20, 2017). Facebook app Replacing Disputed Flags with Related Articles. Facebook. https://about.fb.com/news/2017/12/news-feed-fyi-updates-inour-fight-against-misinformation/

Malmelin, N. and Villi, M. (2016). Audience Community as a Strategic Resource in Media Work. Journalism Practice, 10(5), 589607. https://doi.org/10.1080/17512786.2015.1036903

McDougall, J., Brites, MJ., Couto, MJ. and Lucas, C. (2019). Digital literacy, fake news and education. Culture and Education, 31(2), 203-212. https://doi.org/10.1080/11356405.2019.1603632 
Sixto-García, J., Rodríguez-Vázquez, A-I., \& López-García, X. Sistemas de verificación en medios nativos digitales e implicación de la audiencia en la lucha contra la desinformación en el modelo ibérico.

Mondéjar, D. (May 17, 2019). Verificadores de verificadores, la Unión Europea y Facebook: actores en la lucha contra la desinformación. https://mip.umh.es/blog/2019/05/17/verificadores-union-europea-facebookdesinformacion/

Moreno-Caballud, L. (2014). Cuando cualquiera escribe. Procesos democratizadores de la cultura escrita en la crisis de la Cultura de la Transición española. Journal of Spanish Cultural $\quad$ Studies, $15 \quad$ (1-2), 13-36. https://doi.org/10.1080/14636204.2014.938441

Napoli, P. (2010). Revisiting 'mass communication' and the 'work' of the audience in the new media environment. Media, Culture \& Society, 32(3), 505-516. https://doi.org/10.1177\%2F0163443710361658

Nelson, JL. and Taneja, H. (2018). The small, disloyal fake news audience: The role of audience availability in fake news consumption. New Media \& Society, 20(10), 3720-3737. https://doi.org/10.1177\%2F1461444818758715

Otzen, T. and Manterola, C. (2017). Sampling Techniques on a Population Study. International Journal of Morphology, 35, 227-232. http://dx.doi.org/10.4067/S0717-95022017000100037.

Park, K., Kim, T., Yoon, S., Cha, M. and Jung, K. (2020). BaitWatcher: A lightweight web interface for the detection of incongruent news headlines. In: K. Shu K, S. Wang, D. Lee and H. Liu (Eds.) Fake News, Disinformation and Misinformation in Social Media-Emerging Research Challenges and Opportunities. Springer.

Pennycook, G., Bear, A., Collins, E. and Rand, DG. (2020). The Implied Truth Effect: Attaching Warnings to a Subset of Fake News Headlines Increases Perceived Accuracy of Headlines Without Warnings. Management Science, 66(11), 49444957. https://pubsonline.informs.org/doi/abs/10.1287/mnsc.2019.3478

Pérez, J. (March 12, 2019). Facebook ficha a Newtral y Maldita para combatir las noticias

falsas. https://elpais.com/tecnologia/2019/03/12/actualidad/1552350738 032029.html

Pérez-Escoda, A,. García-Ruiz, R. and Aguaded, I. (2019) Dimensions of digital literacy based on five models of development. Culture and Education, 31(2), 248266. https://doi.org/10.1080/11356405.2019.1603274

Pomerleau, D. and Rao, D. (2016). Fake news challenge stage 1 (FNC-I): Stance detection. http://www.fakenewschallenge.org

Reuters Institute (2019). Digital News Report 2019, Instituto Reuters Institute/ University of Oxford, http://www.digitalnewsreport.org/

Rietjens, S. (2019). Unraveling Disinformation: The Case of Malaysia Airlines Flight MH17. The International Journal of Intelligence, Security, and Public Affairs, 21(3), 195-218. https://doi.org/10.1080/23800992.2019.1695666 
Sixto-García, J., Rodríguez-Vázquez, A-I., \& López-García, X. Sistemas de verificación en medios nativos digitales e implicación de la audiencia en la lucha contra la desinformación en el modelo ibérico.

Shao, C., Ciampaglia, GL., Varol, O., Flammini, A. and Menczer, F. (2018). The spread of fake news by social bots. Nature Communications, 9, 4787-4828. https://www.nature.com/articles/s41467-018-06930-7

Tandoc, E., Lim, Z. and Ling, R. (2018). Defining "Fake News". Digital Journalism, 6(2), 137-153. https://doi.org/10.1080/21670811.2017.1360143

Wardle, C. and Derakhshan, H. (2017). Information disorder toward an interdisciplinary framework for research and policymaking [Pdf File]. Council of Europe

Wigley, C. (2013). Dispelling three myths about Likert scales in communication trait research. Communication Research Reports, 30(4), 366-372. https://doi.org/10.1080/08824096.2013.836937

\section{AUTORES}

José Sixto-García. Es profesor de la Universidade de Santiago de Compostela (USC) y forma parte del grupo de investigación Novos Medios. Sus intereses de investigación incluyen contextos digitales, redes sociales y nuevas narrativas y su impacto en la producción de noticias.

ORCID: https://orcid.org/0000-0002-2988-0975

Google Scholar: https://scholar.google.es/citations?user=65vIOnQAAAAJ\&hl=en

Research Gate: https://www.researchgate.net/profile/Jose-Sixto-Garcia

\section{Ana-Isabel Rodríguez-Vázquez}

Es profesora del Departamento de Ciencias de la Comunicación de la Universidade de Santiago de Compostela. Pertenece al Grupo de Investigación en Estudios Audiovisuales (GEA) de la USC y colabora en proyectos del Grupo Novos Medios. Investiga programación y audiencias.

ORCID: http://orcid.org/0000-0001-7975-1402

Google Scholar: https://scholar.google.es/citations?hl=en\&user=pvFnRVEAAAA]

Research Gate: https://www.researchgate.net/profile/Ana-Rodriquez-Vazquez

\section{Xosé López-García}

Es profesor de periodismo en el Departamento de Ciencias de la Comunicación de la Universidade de Santiago de Compostela. Coordina el grupo de investigación Novos Medios. Sus líneas de investigación incluyen el estudio de los medios digitales e impresos, y el análisis del impacto de la tecnología en la comunicación mediada.

ORCID: http://orcid.org/0000-0002-1873-8260

Google Scholar: https://scholar.google.es/citations?hl=en\&user=omudXhsAAAAJ

Research Gate: https://www.researchgate.net/profile/Xose-Garcia-2 